\title{
Minimal genus problem for pseudo-real Riemann surfaces
}

\author{
Czesław Bagiński and Grzegorz Gromadzki
}

\begin{abstract}
A Riemann surface is said to be pseudo-real if it admits an antiholomorphic automorphism but not an antiholomorphic involution (also known as a symmetry). The importance of such surfaces comes from the fact that in the moduli space of compact Riemann surfaces of given genus, they represent the points with real moduli. Clearly, real surfaces have real moduli. However, as observed by Earle, the converse is not true. Moreover, it was shown by Seppälä that such surfaces are coverings of real surfaces. Here we prove that the latter may always be assumed to be purely imaginary. We also give a characterization of finite groups being groups of automorphisms of pseudo-real Riemann surfaces. Finally, we solve the minimal genus problem for the cyclic case.
\end{abstract}

Mathematics Subject Classification (2000). Primary 30F; Secondary 14H.

Keywords. Pseudo-real Riemann surfaces, Pseudo-symmetric Riemann surfaces, Automorphisms of Riemann surfaces, Anticonformal automorphisms, Symmetric Riemann surfaces, Symmetry of a Riemann surface, Real algebraic curves, Complex algebraic curves with real moduli, Fuchsian and noneuclidean crystallographic groups (NEC).

1. Introduction. The moduli space $\mathbb{M}_{g}$ of complex algebraic curves of genus $g$ is a quasi-projective variety which can be defined in $\mathbb{P}^{\mathrm{n}}(\mathbb{C})$ by polynomials with rational coefficients. Consequently, complex conjugation $\sigma$ in $\mathbb{P}^{n}(\mathbb{C})$ induces an anti-holomorphic involution $\sigma^{*}: \mathbb{M}_{g} \rightarrow \mathbb{M}_{g}$ which maps the class of a complex curve to its conjugate. The points fixed by such a mapping are called complex algebraic curves with real moduli. It is rather obvious that every real algebraic curve has real moduli, though in [4] Earle remarked that the converse is not true. Seppälä [8] showed that the non-real complex algebraic curves with real

C. Baginski was supported by the grant S/W I /3/2008 of Bialystok University of Technology, Bialystok, Poland.

G. Gromadzki was supported by the Research Grant N N201 366436 of the Polish Ministry of Sciences and Higher Education. 
moduli are coverings of real algebraic curves. In [2], Bujalance and Turbek considered such surfaces, which they named pseudo-symmetric surfaces, and in this article, they found all possible groups of automorphisms under the additional assumption that they were also hyperelliptic. More recently, in [3], Bujalance, Conder, and Costa refer to such surfaces as pseudo-real, terminology which we will adopt. In their article they found a bound for the order of their automorphism groups. They also prove that it is attained for infinitely many genera by showing that a large class of surfaces in question comes from the chiral 3-valent regular maps on compact Riemann surfaces.

In the following, we consider a number of results regarding pseudo-real Riemann surfaces. Specifically, we provide a stronger version of the result proved by Seppälä in [8]. We also give a characterization for groups of automorphisms of pseudo-real surfaces. Finally, we solve the minimal genus problem for cyclic groups.

2. Preliminaries. It is well known that the category of smooth, irreducible, projective, complex algebraic curves is equivalent to the category of compact Riemann surfaces and the fact that a complex algebraic curve $\mathcal{C}$ is real means that the corresponding Riemann surface $X=X_{\mathcal{C}}$ admits an anti-holomorphic involution which we call a symmetry (where by anti-holomorphic automorphism we mean a homeomorphism whose local forms composed with the complex conjugation are holomorphic). Moreover, symmetries $\sigma_{1}, \sigma_{2}$, nonconjugate in the group $\operatorname{Aut}^{ \pm}(X)$ of all automorphisms of $X$, give rise to the real forms $\mathcal{C}\left(\sigma_{1}\right), \mathcal{C}\left(\sigma_{2}\right)$, which define real curves with isomorphic complexifications but which are not isomorphic over the reals. Finally, the fact that $\mathcal{C}$ has real moduli means that the corresponding surface $X_{\mathcal{C}}$ admits an anti-holomorphic automorphism. If in addition $X_{\mathcal{C}}$ does not admit a symmetry, it shall be called a pseudo-real Riemann surface, following the paper [3] mentioned above.

We shall study our surfaces and their automorphism groups by means of Fuchsian and non-euclidean crystallographic groups (NEC groups for short). The latter are discrete subgroups of the group $\mathcal{G}$ of isometries of the hyperbolic plane $\mathcal{H}$ with compact orbit spaces. An NEC group is called, respectively, a Fuchsian group or a proper NEC group according to whether it is contained in the subgroup $\mathcal{G}^{+}$of orientation preserving isometries or not. In [7,9], Macbeath and Wilkie associated to every NEC group $\Lambda$ a signature of the form

$$
\left(g ; \pm ;\left[m_{1}, \ldots, m_{r}\right] ;\left\{\left(n_{11}, \ldots, n_{1 s_{1}}\right), \ldots,\left(n_{k 1}, \ldots, n_{k s_{k}}\right)\right\}\right),
$$

which determines the algebraic presentation of $\Lambda$. The numbers $m_{i} \geq 2$ are called the proper periods, the brackets $\left(n_{i 1}, \ldots, n_{i s_{i}}\right)$ are the period cycles, the numbers $n_{i j} \geq 2$ are the link periods and $g \geq 0$ is said to be the orbit genus of $\Lambda$. The orbit space $\mathcal{H} / \Lambda$ is a surface of topological genus $g$, having $k$ boundary components and orientable or not according to the sign being + or -. A Fuchsian group can be regarded as an NEC group with the signature $\left(g ;+;\left[m_{1}, \ldots, m_{r}\right] ;\{-\}\right)$, which shall be abbreviated to $\left(g ; m_{1}, \ldots, m_{r}\right)$; a torsion-free Fuchsian group shall be called a surface Fuchsian group and 
its signature will be denoted by $(g ;-)$. A group with signature (1) has the following generators:

(a) $x_{i}, i=1, \ldots, r$,

(b) $c_{i j}, i=1, \ldots, k ; j=0, \ldots, s_{i}$,

(c) $e_{i}, i=1, \ldots, k$,

(d) $a_{i}, b_{i}, i=1, \ldots, g$, if the sign is + , $d_{i}, i=1, \ldots, g$, if the sign is - ,

and the following relations:

(i) $x_{i}^{m_{i}}=1, i=1, \ldots, r$,

(ii) $c_{i s_{i}}=e_{i}^{-1} c_{i 0} e_{i}, i=1, \ldots, k$,

(iii) $c_{i j-1}^{2}=c_{i j}^{2}=\left(c_{i j-1} c_{i j}\right)^{n_{i j}}=1, i=1, \ldots, k ; j=1, \ldots, s_{i}$,

(iv) $x_{1} \ldots x_{r} e_{1} \ldots e_{k} a_{1} b_{1} a_{1}^{-1} b_{1}^{-1} \ldots a_{g} b_{g} a_{g}^{-1} b_{g}^{-1}=1$, if the sign is + $x_{1} \ldots x_{r} e_{1} \ldots e_{k} d_{1}^{2} \ldots d_{g}^{2}=1$, if the sign is - .

The elements $x_{i}$ are elliptic and represent hyperbolic rotations. The $c_{i j}$ are reflections and $c_{i, j-1}, c_{i, j}$ are said to be consecutive. The $a_{i}, b_{i}$ are hyperbolic translations, the $d_{i}$ are glide reflections and the $e_{i}$ are hyperbolic translations, except for a few special cases when they are elliptic. Every system of generators of an NEC group, satisfying the above relations, will be called a canonical system of generators and, by abuse of language, its elements will be called canonical generators. It is well known that an arbitrary orientation preserving isometry of $\Lambda$ of finite order is either conjugate to a power of a canonical elliptic generator or to a power of the product of two consecutive canonical reflections. Finally each reflection of $\Lambda$ is conjugate to a canonical one.

The hyperbolic area of any fundamental region of the group $\Lambda$, say with the signature (1), depends only on the signature of the group and is given by

$$
\mu(\Lambda)=2 \pi\left(\varepsilon g+k-2+\sum_{i=1}^{r}\left(1-\frac{1}{m_{i}}\right)+\frac{1}{2} \sum_{i=1}^{k} \sum_{j=1}^{s_{i}}\left(1-\frac{1}{n_{i j}}\right)\right),
$$

where $\varepsilon=2$ if the $\operatorname{sign}$ is + and $\varepsilon=1$ otherwise. It is known that an abstract group with the presentation given by the generators $(a)-(d)$ and the relations $(i)-(i v)$ can be realized as an NEC group with the signature (1), if and only if the right hand side of the above expression is positive. Finally, if $\Gamma$ is a finite index subgroup of an NEC group $\Lambda$, then it is an NEC group itself and we have the following formula for the index

$$
[\Lambda: \Gamma]=\mu(\Gamma) / \mu(\Lambda),
$$

known as the Hurwitz-Riemann formula.

Now, by the Riemann uniformization theorem, a compact Riemann surface of genus $g \geq 2$ can be represented as the orbit space $\mathcal{H} / \Gamma$ for some Fuchsian surface group $\Gamma$ with signature $(g ;-)$. Furthermore, a finite group $G$ is a group of automorphisms of a surface given in such a way if it can be represented as the factor group $\Lambda / \Gamma$, where $\Lambda$ is a proper NEC or Fuchsian group according to whether $G$ contains anti-holomorphic automorphisms or not. A symmetry of a Riemann surface $X$ is an anti-holomorphic involution $\sigma$ of $X$, which in terms of NEC groups means that $\langle\sigma\rangle=\Lambda / \Gamma$ for some proper NEC group $\Lambda$. 
3. On groups of automorphisms of pseudo-real Riemann surfaces. First we observe that we have the following generalization of Seppälä's result from [8], where by a purely imaginary real form we mean a real form which has no $\mathbb{R}$-rational points.

Theorem 3.1. A pseudo-real Riemann surface $X$ is a cyclic unbranched covering of degree a power of 2 of a compact Riemann surface $Y$ having a purely imaginary real form.

Proof. Let $X=\mathcal{H} / \Gamma$ and $G=\operatorname{Aut}^{ \pm}(X)=\Lambda / \Gamma$. As $X$ admits an antiholomorphic automorphism but not a symmetry, it follows that $\Lambda$ has glide reflections but no reflections. Consequently, the signature of $\Lambda$ has the form $\left(h ;-;\left[m_{1}, \ldots, m_{r}\right] ;\{-\}\right)$. Now, if the image of $d_{1}$ in $G$ has order $2^{n} m$ for some odd $m$, then $d=d_{1}^{m}$ reverses orientation and, since $X$ has no symmetries, $d^{2} \notin \Gamma$. Therefore, as $d^{2}$ is a hyperbolic translation, $\Delta=\left\langle\Gamma, d^{2}\right\rangle$ has no elliptic elements and so it is a Fuchsian surface group containing $\Gamma$ as a normal subgroup with a cyclic quotient. It follows that for $Y=\mathcal{H} / \Delta$ we have a regular, unbranched, cyclic covering $X \rightarrow Y$ with the group of covering transformations being the subgroup of $G$, generated by the image $\varphi$ of $d^{2}$. Observe also that $\varphi$ has order $2^{n-1}$ and so $\Lambda^{\prime}=\langle\Gamma, d\rangle$ is a subgroup of $\Lambda$ of finite index with the image of $d$ in $\Lambda^{\prime} / \Delta$ representing a fixed point free symmetry of $Y$ and so $Y$ has purely imaginary real form.

Remark 3.2. If, in the configuration from the above proof, $\Lambda$ is a subgroup of an NEC group $\Lambda^{\prime \prime}$ containing a reflection, then there is another construction for a covering of a real curve. Namely, $\Delta=\langle c \Gamma c, \Gamma\rangle$ is a Fuchsian surface group being a subgroup of $\Lambda^{\prime \prime}$ generated by translations and $c$ normalizes $\Delta$. Thus, $Y=\mathcal{H} / \Delta$ is a Riemann surface having a symmetry with fixed points, say represented by $c \Delta$, and unbranched covering $X \rightarrow Y$. Note that this covering neither has to be cyclic nor of degree being a power of 2 .

An action on a pseudo-real Riemann surface admitting an antiholomorphic automorphism is said to be essential. For such actions we have

Theorem 3.3. A finite group $G$ acts as an essential group of automorphisms of a pseudo-real Riemann surface $X$ if and only if it is a non-splitting extension of a group of even order by the cyclic group of order 2. Furthermore a surface $X$ can be chosen to have $G$ as the full group of automorphisms.

Proof. Let $X$ be a non-symmetric Riemann surface and let $G$ be a group of automorphisms of $X$ containing an orientation reversing element $\varphi$. The subgroup $G^{+}=\operatorname{Aut}^{+}(X) \cap G$ of $G$ consisting of orientation preserving elements, is a subgroup of index 2 and, since $X$ has no symmetries, $G$ is a non-splitting extension of $G^{+}$by $\mathrm{Z}_{2}$. Finally, observe that $G^{+}$has even order. Indeed, otherwise $\varphi$ would be an element of order $2 n$ for some odd $n$ and so $\varphi^{n}$ would be a symmetry, contrary to our assumption.

Conversely, let $\alpha: G \rightarrow \mathrm{Z}_{2}$ be a non-splitting epimorphism, let $H$ be its kernel with $h_{1}, \ldots, h_{r}$ being an arbitrary system of generators for $H$, say of orders $m_{1}, \ldots, m_{r}$, and let $d$ be an element of $G \backslash H$. We can assume that 
$r \geq 3$, simply by repeating generators $h_{i}$ if necessary. Let $m_{r+1}$ be the order of $d^{2} h_{1} \ldots h_{r}$.

Assume first that $m_{r+1}=1$. Take a maximal NEC group $\Lambda$ with the signature $\left(1 ;-;\left[m_{1}, \ldots, m_{r}\right] ;\{-\}\right)$, which exists by [5], and let $\theta: \Lambda \rightarrow G$ be an epimorphism defined by $\theta\left(d_{1}\right)=d, \theta\left(x_{i}\right)=h_{i}$ for $i=1, \ldots, r$. Then, since $\alpha$ is non-splitting, $X=\mathcal{H} / \Gamma$ for $\Gamma=\operatorname{ker} \theta$ is a non-symmetric Riemann surface with real moduli, having $G$ as an essential group of automorphisms.

If $m_{r+1} \neq 1$ then, again by [5], there exists a maximal NEC group $\Lambda$ with the signature $\left(1 ;-;\left[m_{1}, \ldots, m_{r}, m_{r+1}\right] ;\{-\}\right)$. Let $\theta: \Lambda \rightarrow G$ be an epimorphism defined by $\theta\left(d_{1}\right)=d, \theta\left(x_{i}\right)=h_{i}$ for $i=1, \ldots, r$ and let $\theta\left(x_{r+1}\right)=$ $\left(d^{2} h_{1} \ldots h_{r}\right)^{-1}$. Again, as $\alpha$ is non-splitting, $X=\mathcal{H} / \Gamma$ for $\Gamma=\operatorname{ker} \theta$ is a nonsymmetric Riemann surface with real moduli, having $G$ as an essential group of automorphisms.

4. Some technical results concerning NEC groups. In this section we shall prove three technical lemmas concerning essential actions on pseudo-real Riemann surfaces. The first one can be seen as a generalization of the Proposition 5.2 from [3].

Lemma 4.1. Let $\Lambda$ be an NEC group with the signature $(1 ;-;[k, l] ;\{-\})$, where $k \neq l$. Then there exists an epimorphism $\theta: \Lambda \rightarrow G$ onto a finite group $G$, defining an essential action of $G$ on a pseudo-real Riemann surface, if and only if $G$ is a non-splitting extension of its subgroup $H$ of index $2, G$ is generated by two elements $x, d$ such that $x$ and $d^{2} x$ have orders $k$ and $l$, respectively, $d \notin H$ and the mapping $x \mapsto x^{-1}, d \mapsto d^{-1}$ does not induce an automorphism of $G$. Furthermore such a group $G$ is necessarily the full group of automorphisms of a pseudo-real Riemann surface on which it acts.

Proof. Let $X=\mathcal{H} / \Gamma$ for $\Gamma=\operatorname{ker} \theta$. Observe first that $H=\theta\left(\Lambda^{+}\right)$is an index 2 subgroup of $G$ else ker $\theta$ would contain an orientation reversing isometry and so $X$ would be non-orientable. Furthermore, the embedding $H \leq G$ is nonsplitting since an element of order 2 in $G \backslash H$ would represent a symmetry of $X$. Now, since $\theta$ preserves the orders of elliptic elements, the images under $\theta$ of $x_{1}$ and $x_{2}=\left(d_{1}^{2} x_{1}\right)^{-1}$ are elements of orders $k$ and $l$. As $\Gamma$ is a Fuchsian group, $d=\theta\left(d_{1}\right) \notin H$ and so for the necessity it is sufficient to show that for $x=\theta\left(x_{1}\right)$ and $d=\theta\left(d_{1}\right)$, the mapping $x \mapsto x^{-1}, d \mapsto d^{-1}$ does not induce an automorphism of $G$. To see this, first observe that by $[1,5]$, there is an NEC group $\Lambda^{\prime}$, with the unique signature $(0 ;+;[2] ;\{(k, l)\})$, containing $\Lambda$ as a subgroup of index 2. After reductions, $\Lambda^{\prime}$ has the presentation

$$
\left\langle x_{1}^{\prime}, c_{0}^{\prime}, c_{1}^{\prime} \mid c_{0}^{\prime 2}, c_{1}^{\prime 2}, x_{1}^{\prime 2},\left(c_{0}^{\prime} c_{1}^{\prime}\right)^{k},\left(c_{1}^{\prime} x_{1}^{\prime} c_{0}^{\prime} x_{1}^{\prime}\right)^{l}\right\rangle .
$$

Now, we shall find how the elements of a canonical system of generators for $\Lambda$ can be expressed by those for $\Lambda^{\prime}$. For, let $\eta: \Lambda^{\prime} \rightarrow \Lambda^{\prime} / \Lambda=\mathrm{Z}_{2}=\langle a\rangle$ be the canonical projection. Then $\eta\left(c_{i}^{\prime}\right)=a$ since $\Lambda$ has no reflections. Now $x_{1}^{\prime}$ and $e_{1}^{\prime}$ are the only orientation preserving canonical generators of $\Lambda^{\prime}$ and $\theta\left(x_{1}^{\prime}\right)=\theta\left(e_{1}^{\prime}\right)$. So since $\Lambda$ contains a glide reflection, $\theta\left(x_{1}^{\prime}\right)=a$ and therefore

$$
x_{1}=c_{0}^{\prime} c_{1}^{\prime}, x_{2}=c_{1}^{\prime} x_{1}^{\prime} c_{0}^{\prime} x_{1}^{\prime}, d_{1}=x_{1}^{\prime} c_{0}^{\prime},
$$


are elements of $\Lambda$. But below, in (3), we shall show that they generate a normal subgroup $K$ of $\Lambda^{\prime}$. Furthermore $\Lambda^{\prime} / K=\mathrm{Z}_{2}$ and finally it is easy to check that they satisfy canonical relations for $\Lambda$. So $\Lambda=K$ and therefore the above elements can be chosen for a system of canonical generators of $\Lambda$. Now for the action of $\Lambda^{\prime}$ on $\Lambda$, we have:

$$
c_{0}^{\prime} x_{1} c_{0}^{\prime}=x_{1}^{-1}, c_{0}^{\prime} x_{2} c_{0}^{\prime}=x_{1} x_{2}^{-1} x_{1}^{-1}, c_{0}^{\prime} d_{1} c_{0}^{\prime}=d_{1}^{-1}
$$

and so $\Gamma$ is a normal subgroup of $\Lambda^{\prime}$ if and only if

$$
x \mapsto x^{-1}, \quad d \mapsto d^{-1}
$$

induces an automorphism of $G$. So the assertion follows, since in the last case $\operatorname{Aut}^{ \pm}(X)=\Lambda^{\prime} / \Gamma$ and so $X$ has a symmetry, represented for example by $c_{0} \Gamma$, which contradicts our assumption on $X$.

Conversely, for an NEC group $\Lambda$ with signature $(1 ;-;[k, l] ;\{-\})$ and $(G, H)$ being the pair in question, the mapping $\theta\left(x_{1}\right)=x, \theta\left(d_{1}\right)=d$ and $\theta\left(x_{2}\right)=$ $\left(d^{2} x\right)^{-1}$ induces an epimorphism $\theta: \Lambda \rightarrow G$, defining an essential action of $G$ on the Riemann surface $X=\mathcal{H} / \Gamma$, where $\Gamma=\operatorname{ker} \theta$. Now $G$ is the full group of automorphisms, since otherwise $\Gamma$ would be a normal subgroup of an NEC group $\Lambda^{\prime}$ with signature $(0 ;+;[2] ;\{(k, l)\})$ and so, by the previous part of the proof, the mapping $x \mapsto x^{-1}, d \mapsto d^{-1}$ would define an automorphism of $G$, contrary to our assumption. Finally, since $G$ is a non-splitting extension of $H$, $G$ contains no symmetries and, consequently, $X$ is pseudo-real.

The last part of the lemma follows from the fact that the signature of $\Lambda^{\prime}$ containing $\Lambda$ is unique and we have shown that $\Gamma$ is normal in $\Lambda^{\prime}$ just if and only if the corresponding surface $X=\mathcal{H} / \Gamma$ is not pseudo-real.

Lemma 4.2. Let $\Lambda$ be an NEC group with the signature $(1 ;-;[k, k] ;\{-\})$, where $k>2$. Then there is an epimorphism $\theta: \Lambda \rightarrow G$ onto a finite group $G$, defining an essential action of $G$ on a pseudo-real Riemann surface, if and only if $G$ is a non-splitting extension of its subgroup $H$ of index $2, G$ is generated by two elements $x, d$ such that $x$ and $d^{2} x$ have order $k, d \notin H$ and neither the map $x \mapsto x^{-1}, d \mapsto d^{-1}$ nor $x \mapsto x^{-1} d^{-2}, d \mapsto d$ induces an automorphism of $G$. Furthermore such a group $G$ is necessarily the full group of automorphisms of a pseudo-real Riemann surface on which it acts.

Proof. Let $X=\mathcal{H} / \Gamma$ for $\Gamma=\operatorname{ker} \theta$. As in the previous lemma, $H=\theta\left(\Lambda^{+}\right)$is a subgroup of index 2 and the embedding $H \leq G$ is non-splitting. Now, since $\theta$ preserves the orders of elliptic elements, the images of $x_{2}$ and $x_{1}=\left(x_{2} d_{1}^{2}\right)^{-1}$ are elements of order $k$ and, as $\Gamma$ is a Fuchsian group, $d=\theta\left(d_{1}\right) \notin H$. To prove the necessary condition it is sufficient to show that none of the maps in the lemma induces an automorphism of $G$. By $[1,5]$, an NEC group $\Lambda^{\prime}$, containing $\Lambda$ as a normal subgroup, must have one of the signatures $(0 ;+;[2] ;\{(k, k)\})$, $(0 ;+;[2, k] ;\{(-)\})$ or $(0 ;+;[-] ;\{(2,2,2, k)\})$ and the index $\left[\Lambda^{\prime}: \Lambda\right]$ equals 2,2 and 4 , respectively. In the first case, repeating word by word the proof of the previous lemma for $k=l$, we obtain the assertion concerning the first mapping. Now let $\Lambda^{\prime}$ be an NEC group with signature $(0 ;+;[2, k] ;\{(-)\})$. Then, 
after obvious reductions, it has presentation

$$
\left\langle x_{1}^{\prime}, x_{2}^{\prime}, c_{0}^{\prime} \mid x_{1}^{\prime 2}, x_{2}^{\prime k}, c_{0}^{\prime 2},\left(x_{1}^{\prime} x_{2}^{\prime}\right) c_{0}^{\prime}\left(x_{1}^{\prime} x_{2}^{\prime}\right)^{-1} c_{0}^{\prime}\right\rangle .
$$

As in the previous lemma we can argue that

$$
x_{1}=x_{1}^{\prime} x_{2}^{\prime} x_{1}^{\prime}, \quad x_{2}=x_{2}^{\prime}, \quad d_{1}=c_{0}^{\prime}\left(x_{1}^{\prime} x_{2}^{\prime}\right)^{-1}
$$

can be chosen as a canonical system of generators for $\Lambda$.

Furthermore,

$$
x_{1}^{\prime} x_{2} x_{1}^{\prime}=d_{1}^{-2} x_{2}^{-1}, \quad x_{1}^{\prime} d_{1} x_{1}^{\prime}=x_{2} d_{1} x_{2}^{-1}
$$

and so $\Gamma$ is a normal subgroup of $\Lambda^{\prime}$ if and only if for $x=\theta\left(x_{2}\right), d=\theta\left(d_{1}\right)$, the map $x \mapsto d^{-2} x^{-1}, d \mapsto x d x^{-1}$ induces an automorphism of $G$. But the last is equivalent that the map

$$
x \mapsto x^{-1} d^{-2}, \quad d \mapsto d
$$

induces an automorphism of $G$ also and hence the assertion follows, since the latter is equivalent to $\Lambda^{\prime} / \Gamma \subseteq \operatorname{Aut}^{ \pm}(X)$, which in turn means that $X$ has a symmetry, represented for example by $c_{0} \Gamma$, contrary to our assumption on $X$.

Conversely, for an NEC group $\Lambda$ with the signature $(1 ;-;[k, l] ;\{-\})$ and the pair $(G, H)$ in question, the mapping $\theta\left(x_{2}\right)=x, \theta\left(d_{1}\right)=d$ and $\theta\left(x_{1}\right)=\left(x d^{2}\right)^{-1}$ induces an epimorphism $\theta: \Lambda \rightarrow G$, defining a Riemann surface $X=\mathcal{H} / \Gamma$, where $\Gamma=\operatorname{ker} \theta$, with the group $G$ being an essential group of automorphisms. Also, $G$ has no symmetries as it is a non-splitting extension of $H$. Now $\Gamma$ is not a normal subgroup of an NEC group with the signature $(0 ;+;[2] ;\{(k, k)\})$, since otherwise the map $x \mapsto x^{-1}, d \mapsto d^{-1}$ would define an automorphism of $G$. Similarly, $\Gamma$ is not a normal subgroup of an NEC group with the signature $(0 ;+;[2, k] ;\{(-)\})$, since otherwise $x \mapsto x^{-1} d^{-2}, d \mapsto d$ would define such an automorphism. Furthermore, $\Gamma$ cannot be a normal subgroup of an NEC group with the signature $(0 ;+;[-] ;\{(2,2,2, k)\})$, since the last contains a group with the signature $(0 ;+;[2, k] ;\{(-)\})$, which in turn contains $\Lambda$. Therefore $G$ is the full group of automorphisms of $X$, being a pseudo-real Riemann surface, and the proof is complete.

Lemma 4.3. Let $\Lambda$ be an NEC group with signature $(2 ;-;[k] ;\{-\})$. Then there is an epimorphism $\theta: \Lambda \rightarrow G$ onto a finite group $G$, defining an essential action of $G$ on a pseudo-real Riemann surface, if and only if $G$ is a non-splitting extension of its subgroup $H$ of index $2, G$ is generated by two elements $x, y$ such that $x^{2} y^{2}$ has order $k, x, y \notin H$ and the mapping $x \mapsto y^{-2} x^{-1}, y \mapsto y^{-1} x^{-2}$ does not induce an automorphism of $G$. Furthermore such a group $G$ is necessarily the full group of automorphisms of a pseudo-real Riemann surface on which it acts.

Proof. Let, as before, $X=\mathcal{H} / \Gamma$ for $\Gamma=\operatorname{ker} \theta$ and observe that $H=\theta\left(\Lambda^{+}\right)$is a subgroup of index 2 , with the embedding $H \leq G$ being non-splitting. Moreover, the image of a canonical elliptic element of $\Lambda$ under $\theta$ is an element of order $k$, as $\operatorname{ker} \theta$ is torsion free. Now, since $\Gamma$ is a Fuchsian group, $x=\theta\left(d_{1}\right)$ and $y=\theta\left(d_{2}\right)$ are not in $H$ and, as $x_{1} d_{1}^{2} d_{2}^{2}=1$, they generate $G$. Hence, for the necessity, it is sufficient to show, that for $x=\theta\left(d_{1}\right)$ and $y=\theta\left(d_{2}\right)$, the mapping 
in question does not induce an automorphism of $G$. To see this, first observe that, by $[1,5]$, there is an NEC group $\Lambda^{\prime}$ with the signature $(0 ;+;[2,2] ;\{(k)\})$, containing $\Lambda$ as a subgroup of index 2 , and this signature is unique. Now, after reductions, $\Lambda^{\prime}$ has the presentation

$$
\left\langle x_{1}^{\prime}, x_{2}^{\prime}, c_{0}^{\prime} \mid x_{1}^{\prime 2}, x_{2}^{\prime 2}, c_{0}^{\prime 2},\left(c_{0}^{\prime} x_{1}^{\prime} x_{2}^{\prime} c_{0}^{\prime} x_{2}^{\prime} x_{1}^{\prime}\right)^{k}\right\rangle
$$

and as in Lemma 4.1 we argue that

$$
d_{1}=x_{1}^{\prime} x_{2}^{\prime}, \quad d_{2}=x_{2}^{\prime} x_{1}^{\prime} c_{0}^{\prime}, \quad x_{1}=c_{0}^{\prime} x_{1}^{\prime} x_{2}^{\prime} c_{0}^{\prime} x_{2}^{\prime} x_{1}^{\prime}
$$

can be chosen as a canonical system of generators of $\Lambda$. Furthermore, for the action of $\Lambda^{\prime}$ on $\Lambda$, we have:

$$
c_{0}^{\prime} d_{1} c_{0}^{\prime}=d_{2}^{-2} d_{1}^{-1}, \quad c_{0}^{\prime} d_{2} c_{0}^{\prime}=d_{2}^{-1} d_{1}^{-2}
$$

and so $\Gamma$ is a normal subgroup of $\Lambda^{\prime}$ if and only if the map

$$
x \mapsto y^{-2} x^{-1}, \quad y \mapsto y^{-1} x^{-2}
$$

induces an automorphism of $G$. So our assertion follows, since in the last case $\operatorname{Aut}^{ \pm}(X)=\Lambda^{\prime} / \Gamma$ and $X$ has a symmetry represented, for example, by $c_{0} \Gamma$.

Conversely, given an NEC group $\Lambda$ with the signature $(2 ;-;[k] ;\{-\})$ and the couple $(G, H)$ in question, the mapping $\theta\left(d_{1}\right)=x, \theta\left(d_{2}\right)=y, \theta\left(x_{1}\right)=$ $y^{-2} x^{-2}$ induces an epimorphism $\theta: \Lambda \rightarrow G$, defining a Riemann surface with $G$ as an essential group of its automorphisms. As in Lemmas 4.1 and 4.2 we argue that $X$ is pseudo-real and in such case $G=\operatorname{Aut}(X)$.

5. Some elementary number theory. Theorem 3.3 shows that a cyclic group is an essential group of automorphisms of a pseudo-real Riemann surface if and only if it has order $4 N$. In the next section we shall find the minimal genus of a pseudo-real Riemann surface for such an action. To do this, we shall need the following lemma from elementary number theory.

Lemma 5.1. Let $N$ be an arbitrary positive integer, let first $p$ be an odd prime dividing $N$ and let $G=\left\langle a \mid a^{4 N}\right\rangle$. Then there exist elements $x, y \in G-\left\langle a^{2}\right\rangle$ satisfying the following three conditions:

(a) $G=\langle x, y\rangle$,

(b) The element $x^{2} y^{2}$ has order $p$,

(c) There does not exist an automorphism $\alpha$ of $G$ such that

$$
\left\{\begin{array}{l}
\alpha(x)=x^{-1} y^{-2}, \\
\alpha(y)=y^{-1} x^{-2} .
\end{array}\right.
$$

Moreover such a pair exists for $p=2^{m}$, if and only if $N=2^{s} n$ with $n$ odd, $s \geq 3$ and $2 \leq m \leq s$.

Proof. Let $p$ be an odd prime and suppose $N=p n$. We put

$$
x=a, \quad y=a^{-1+4 k n},
$$

where $k \in\{1,2, \ldots, p-1\}$ and $2 k n \not \equiv 1(\bmod p)$. Then obviously, $x, y \in$ $G-\left\langle a^{2}\right\rangle, G=\langle x\rangle=\langle x, y\rangle$ and $x^{2} y^{2}=\left(a^{4 k n}\right)^{2}$ has order $p$. 
Now, suppose that $\alpha$ is an automorphism of $G$ such that $\alpha(x)=x^{-1} y^{-2}=$ $a^{1-8 k n}$. Then for every $g \in G, \alpha(g)=g^{1-8 k n}$, as $x=a$ and $G$ is cyclic. Hence $\alpha(y)=y^{-1} x^{-2}$ if and only if $\alpha\left(a^{-1+4 k n}\right)=a^{-1-4 k n}$ which is equivalent to the congruence

$$
(-1+4 k n)(1-8 k n) \equiv-1-4 k n \quad(\bmod 4 p n),
$$

which in turn means

$$
16 k n(1-2 k n) \equiv 0 \quad(\bmod 4 p n) .
$$

However if $p$ divides $n$ then the last equality is not possible while in the other case it is true just for one value of $k$, namely the value for which $2 k n \equiv 1$ $(\bmod p)$. Therefore $\alpha(y) \neq y^{-1} x^{-2}$ by the definition of $y$.

Now assume that $p=2^{m}, m \geq 1$ and $N=2^{s} n$, where $n$ is odd. In particular $|G|=2^{s+2} n$. Write $\langle a\rangle=\langle b\rangle \oplus\langle c\rangle$, where $b=a^{n}, c=a^{2^{s+2}}$. If for $x, y \in\langle a\rangle, x y$ has order $2^{m+1}$ and $x=x_{1} x_{2}, y=y_{1} y_{2}$, where $x_{1}, y_{1} \in\langle b\rangle$, $x_{2}, y_{2} \in\langle c\rangle$, then $x y \in\langle b\rangle$ and so we have $y_{2}=x_{2}^{-1}$. Note also that $x_{1}, y_{1} \notin$ $\left\langle b^{2}\right\rangle$, since otherwise at least one of these elements would belong to $\left\langle a^{2}\right\rangle$. Thus $\left\langle x_{1}\right\rangle=\langle b\rangle=\left\langle y_{1}\right\rangle$ which implies $x y=x_{1} y_{1} \in\left\langle b^{2}\right\rangle$ and then $m \leq s$. Moreover $G=\langle x, y\rangle=\left\langle x_{1}, x_{2}, y_{1}, y_{2}\right\rangle=\left\langle x_{1}, x_{2}\right\rangle=\langle x\rangle$ and similarly $G=\langle y\rangle$. Therefore we may assume that $x=a$ and, because elements of order $2^{m+1}$ are exactly elements of the form $a^{2^{s-m+1} k n}$, where $k$ is odd, we get $y=a^{-1+2^{s-m+1} k n}$. Hence

$$
x^{-1} y^{-2}=a^{1-2^{s-m+2} k n}, \quad y^{-1} x^{-2}=a^{-1-2^{s-m+1} k n} .
$$

If we suppose that $\alpha$ is an automorphism of $G$ such that $\alpha(x)=x^{-1} y^{-2}$, then for every $g \in G$

$$
\alpha(g)=g^{1-2^{s-m+2} k n} .
$$

So, $\alpha(y)=y^{-1} x^{-2}$ if and only if $\alpha\left(a^{-1+2^{s-m+1} k n}\right)=a^{-1-2^{s-m+1} k n}$ which is equivalent to the congruence

$$
\left(-1+2^{s-m+1} k n\right)\left(1-2^{s-m+2} k n\right) \equiv-1-2^{s-m+1} k n \quad\left(\bmod 2^{s+2} n\right) .
$$

This means

$$
2^{s-m+3} k n\left(1-2^{s-m} k n\right) \equiv 0 \quad\left(\bmod 2^{s+2} n\right)
$$

which in turn is equivalent to

$$
2\left(1-2^{s-m} k n\right) \equiv 0 \quad\left(\bmod 2^{m}\right) .
$$

If $m=1$, then this congruence holds true and hence a pair $x, y$ of elements satisfying all three conditions does not exist. The same happens when $s=m=2$.

If $s>m \geq 2$ then this congruence does not hold which means that the elements $x$ and $y$ satisfy all three conditions.

If $s=m>2$ then we can find $k$ such that $k n \equiv-1\left(\bmod 2^{m}\right)$ which gives

$$
2\left(1-2^{s-m} k n\right) \equiv 4 \not \equiv 0 \quad\left(\bmod 2^{m}\right) .
$$

Hence, again, for such a $k$ the elements $x, y$ satisfy our conditions. 
Remark 5.2. A cyclic group $G$ of order $2^{t}$, where $2 \leq t \leq 4$, does not contain elements $x, y$ satisfying the three conditions of the above Lemma for any $p>1$.

6. Minimal genus problem. To finish, we state and prove the principal result of the paper concerning the minimal genus problem for cyclic groups of automorphisms of pseudo-real Riemann surfaces. We already know, from Theorem 3.3, that such a group has order $4 N$.

Theorem 6.1. The minimal genus of a pseudo-real Riemann surface, admitting a cyclic group of order $4 N$ as an essential group of automorphisms, equals

$$
\begin{array}{ll}
2 N & \text { if } N=1,2,4, \\
3 N / 2+1 & \text { if } 8 \mid N \text { and } 3 \text { does not divide } N, \\
2(p-1) N / p+1 & \text { otherwise, }
\end{array}
$$

where $p$ is the smallest nontrivial odd prime divisor of $N$.

Proof. Let $G=\left\langle a \mid a^{4 N}\right\rangle$ be a group of automorphisms of a pseudo-real Riemann surface $X=\mathcal{H} / \Gamma$, let $G=\Lambda / \Gamma$ and let $\theta: \Lambda \rightarrow G$ be the canonical projection. Clearly the minimal genus for $X$ will correspond to an NEC group $\Lambda$ with the smallest possible area. Since $X$ has no symmetries, $\Lambda$ has no reflections and so it has signature of the form $\left(h ;-;\left[m_{1}, \ldots, m_{r}\right] ;\{-\}\right)$. Suppose first that $N \neq 1,2,4$.

If $h \geq 3$ or $h=2, r \geq 2$ or $h=1, r \geq 4$ then $\mu(\Lambda) \geq 2 \pi$. For $h=1$ and $r=1$ we have that $\mu(\Lambda)<0$ while $r \neq 2$ by Lemmas 4.1 and 4.2 . Therefore $\mu(\Lambda) \geq 2 \pi$ and so $g \geq 2 N+1$ except when $\Lambda$ has signature

$$
\left(1 ;-;\left[m_{1}, m_{2}, m_{3}\right] ;\{-\}\right)
$$

or

$$
(2 ;-;[m] ;\{-\}) \text {. }
$$

Consider first the signature (5) and observe that the images, under $\theta$, of the canonical elliptic generators of $\Lambda$ cannot generate $G$ since in such case $d_{1} \omega\left(x_{1}, x_{2}, x_{3}\right)$ would belong to $\Gamma=\operatorname{ker} \theta$ for some word $\omega$ and so $\Gamma$ would be a proper NEC group, contrary to the assumption that it uniformizes a Riemann surface. So, since $x_{1} x_{2} x_{3} d_{1}^{2}=1$, the images of $x_{1}, x_{2}, x_{3}$ generate the subgroup of order $2 N$. Therefore one of the periods is divisible by $p$ and one of them is divisible by 4 when $N$ is even but not a power of 2 . If $N$ is a power of 2 then one of the periods is divisible by $2 N$. Finally if $N$ is odd then a priori the minimal area for $\Lambda$ we obtain when it has signature $(1 ;-;[2,2, p] ;\{-\})$. However, here $d_{1}^{2} x_{1} x_{2} x_{3}=1$ and so $\theta\left(d_{1}\right)^{2}=\theta\left(x_{3}\right)^{-1}$. Hence $\theta\left(d_{1}\right)$ would have order $2 p$ and therefore $\theta$ would not be an epimorphism.

We shall now show that the smaller, than above, area and hence smaller genus, can be achieved for the signature (6). For, if $N$ is a multiple of 8 then an NEC group with signature (6) with the smallest possible area occurs, by Lemmas 4.3 and 5.1 , for $m=4$ if $p \neq 3$ and for $m=3$ if $p=3$. So the minimal genera in these cases are equal to $3 N / 2+1$ or $4 N / 3+1=2(p-1) N / p+1$, respectively. Next, if 8 does not divide $N$ then again by Lemmas 4.3 and 5.1 we obtain the minimum genus from the signature (6) for $m=p$, i.e., the minimum genus in this case is $2(p-1) N / p+1$. Observe finally that the genera 
obtained here are smaller than the ones which can be obtained from the group with signature (5), considered in the previous paragraph.

The cyclic groups of order $4 N$ for $N=1,2,4$ must be considered separately as by Lemma 4.3 and Remark 5.2 the signature (6) does not provide an action of $\mathrm{Z}_{4 N}$ on a pseudo-real Riemann surface in each of these cases. Thus we have to consider an NEC group $\Lambda$ with signature (5). As before, the images of $x_{1}, x_{2}, x_{3}$ generate the group of order $2 N$ and so the image of some $x_{i}$ must be an element of order $2 N$, which means that $m_{i}=2 N$ for some $i$. Therefore, the signature (5) with $m_{1}=m_{2}=2, m_{3}=2 N$, is the signature of an NEC group with the smallest possible area in this case. Now, by $[1,5]$, given $N$ there is a maximal NEC groups $\Lambda$ with signature $(1 ;-;[2,2,2 N] ;\{-\})$ and so considering an epimorphism $\theta: \Lambda \rightarrow \mathrm{Z}_{4 N}=\langle a\rangle$ defined by

$$
\theta\left(d_{1}\right)=a, \theta\left(x_{1}\right)=\theta\left(x_{2}\right)=a^{2 N}, \quad \theta\left(x_{3}\right)=a^{-2}
$$

we obtain the minimal genera for $N=1,2$ and 4 . Observe that $\mathrm{Z}_{4 N}$ for the resulting surface is the full group of automorphisms.

Remark 6.2. As was remarked at the end of the proof of Theorem 6.1, a pseudoreal Riemann surface $X$ of the minimal genus with a group of automorphism $\mathrm{Z}_{4 N}$ for $N=1,2,4$ can be chosen in such a way that $\mathrm{Z}_{4 N}=\operatorname{Aut}(X)$. Now for $N \neq 1,2,4$ and any such surface $X$ we have $\mathrm{Z}_{4 N}=\operatorname{Aut}(X)$, due to the last part of the Lemma 4.3, since the minimal genus action here can be obtained only from an NEC group $\Lambda$ with signature $(2 ;-;[m] ;\{-\})$.

Remark 6.3. In [6], Etayo found the minimal genus for orientable Riemann surfaces which admit an orientation reversing automorphism of given order. His article however, does not solve the minimal genus problem for cyclic groups of automorphisms of pseudo-real Riemann surfaces since, in most cases, cyclic groups of automorphisms are not the full groups of automorphisms and Etayo's surfaces admit symmetries, i.e., they are not pseudo-real. The reader can check that indeed the values in our Theorem 6.1 are greater than the ones given in Theorem 5 of $[6]$.

Acknowledgements. The authors are very grateful to the referee for his (her) vigilant reading of the paper, very helpful comments, for pointing out an error in the first version of Theorem 6.1 and remarking some inaccuracies still in the revised version. Our gratitude is also directed to Professor Ernst-Ulrich Gekeler, the Managing Editor, for pointing out linguistic defects of the paper, for allowing its resubmission and finally to Aaron Wootton for the reading of the final version of the paper and linguistic improvements.

Open Access. This article is distributed under the terms of the Creative Commons Attribution Noncommercial License which permits any noncommercial use, distribution, and reproduction in any medium, provided the original author(s) and source are credited. 


\section{References}

[1] E. Bujalance, Normal NEC signatures, Illinois J. Math. 26 (1982), 519-530.

[2] E. Bujalance And P. Turbek, Asymmetric and pseudo-symmetric hyperelliptic surfaces, Manuscripta Math. 108 (2002), 1-11.

[3] E. Bujalance, M. Conder, and A. F. Costa, Pseudo-real Riemann surfaces and chiral regular maps, Trans. Amer. Math. Soc. 362 (2010), 3365-3376.

[4] C. Earle, On moduli of closed Riemann surfaces with symmetries, Advances in the theory of Riemann surfaces, Annals of Mathematics Studies 66 (1971), 119-130.

[5] J. L. Estévez And M. IzQuierdo, Non-normal pairs of noneuclidean crystallographic groups, Bull. London Math. Soc 38 (2006), 113-123.

[6] J. J. Etayo Gordejuela, Non-orientable automorphisms of Riemann surfaces, Arch. Math. 45 (1985), 374-384.

[7] A. M. Macbeath, The classification of non-euclidean crystallographic groups, Can. J. Math. 19 (1967), 1192-1205.

[8] M. SEpp ̈̈цÄ, Complex algebraic curves with real moduli, J. reine angew. Math. 387 (1988), 209-220.

[9] H. C. Wilkie, On non-euclidean crystallographic groups, Math. Z. 97 (1966), 87-102.

\section{Czeseaw Bagiński}

Faculty of Computer Science,

Białystok University of Technology,

Wiejska 45, 15-351 Bialystok, Poland

e-mail: c.baginski@pb.edu.pl

Grzegorz Gromadzki

Institute of Mathematics, University of Gdańsk, Wita Stwosza 57, 80-952 Gdańsk, Poland

e-mail: greggrom@math.univ.gda.pl

Received: 11 March 2010

Revised: 21 July 2010 